Research Article

\title{
Fault Detection of Aircraft Control System Based on Negative Selection Algorithm
}

\author{
Jie Chen, Senyao Chen (D), Cunbao Ma, Zhengdong Jing, and Qingshan Xu \\ School of Civil Aviation, Northwestern Polytechnical University, Xi'an 710072, China \\ Correspondence should be addressed to Senyao Chen; 782850331@qq.com
}

Received 30 July 2020; Revised 24 September 2020; Accepted 14 October 2020; Published 4 November 2020

Academic Editor: Paolo Castaldi

Copyright @ 2020 Jie Chen et al. This is an open access article distributed under the Creative Commons Attribution License, which permits unrestricted use, distribution, and reproduction in any medium, provided the original work is properly cited.

\begin{abstract}
The aircraft control system controls the whole flight movement process. Its fault detection can assist the aircraft PHM system in making decisions and completing the targeted maintenance, which is of great significance to improve the safety and reliability of the aircraft. In this paper, by taking advantage of the strong leaning and intelligent recognition ability and the characteristic of less information required in the negative selection artificial immune system, a fault detection method is proposed for aircraft control system based on negative selection algorithm. Basically, after extracting the fault characteristics from the aircraft flight parameters, the negative selection module is utilized to generate fault detectors to monitor the aircraft control system. Afterward, the hypothesis test is introduced to evaluate the detector coverage more efficiently, and the detector cover area is optimized by applying geometric mathematics in the optimization of the detector center position and radius. The method is verified by simulation of a certain aircraft control system, and the results show that it has a good detection effect on the system faults.
\end{abstract}

\section{Introduction}

The aircraft control system completes flight attitude and trajectory control and achieves specific flight actions, which is closely linked with flight safety. In recent years, with the rapid development of aviation technology, the performance of control system has been significantly improved. But as the airborne system with the highest safety level requirements, the sudden failure of this system is very likely to cause harm to pilots and passengers, leading to disastrous consequences in the face of complex high-altitude flight conditions [1]. Therefore, it is crucial to establish an efficient fault detection method to cover more faults to ensure the 0 safety and reliability of aircraft.

In view of this, many scholars in the world are committed to solving the problem of aircraft control system fault detection. Xu and Sun [2] optimized the traditional fault detection method by adopting the neural networks to simplify the detection steps of the expert system. Duan and Zhang [3] took the system modeling and FMECA as the theoretical basis to realize the detection, and Cheng [4] analyzed the flight parameter data from QAR and found the flap extracting and retracting time can be applied to realize the flap system fault detection. But in general, these traditional modelbased fault detection methods are required a large number of observers; the diagnosis process is so complicated and inefficient. Furthermore, the fault detection coverage cannot be guaranteed by the sole feature parameter, while these problems can be solved well by the artificial immune system.

Artificial immune system is a new intelligent diagnosis system inspired by biological immune system, which is another intelligent system emerged after neural networks and genetic algorithms [5]. It has many enlightening special functions for practical engineering problems, such as pattern recognition, memory, and strong learning [6]. In 1986, the artificial immune system began to be studied and applied to fault detection. And relying on its powerful information processing capabilities, it was gradually widely utilized in the field of fault diagnosis [7]. Laurentys et al. [8] proposed a dynamic system fault detection method based on artificial immune system, while Ghosh and Srinivasan [9] took it in the field of fault diagnosis and process monitoring, and Diego 
et al. [10] further applied it on the spacecraft fault diagnosis. The artificial immune system is also widely made use of in aviation. Sha [11] took the artificial immune system into the fault detection of the engine numerical control system; the immune theory was utilized to establish an output estimation module which reduced the error between the actual sensor and the model output. However, for complex systems, such as aircraft control systems, this method is cumbersome required too many control parameters, leading to the low fault detection accuracy.

In recent years, a variety of algorithms such as negative selection, clonal selection, and immune genetics have been produced based on the principle of artificial immune system. Among them, negative selection algorithm has played an important role in the field of fault detection. Compared with other intelligent algorithms like neural networks and clustering which need mass of fault data to achieve high fault recognition accuracy, negative selection generates fault detectors from large amount of flight normal data which is more suitable for the current development trend. Li [12] and Zhang [13] applied it in the detection of aero engine wear faults and aircraft electromechanical system faults, respectively, adopting negative selection theory to generate initial detectors and clonal selection theory to select mature detectors, so as to achieve the corresponding system failure detection. However, the traditional negative selection will generate a large number of redundant detectors during the initial detector generation stage, resulting in greatly reduced selection efficiency and accuracy of mature detectors, which in turn will affect the detection effect. What is more, the applications of artificial immune systems are less in the field of aircraft control systems.

In response to the above problems, the method is established to detect fault in the aircraft control system based on negative selection in this paper. The center position and radius of the fault detector are optimized to improve the detector coverage, as well as the hypothesis testing is introduced during the generation of the detector as the control condition for the end of the algorithm, thereby reducing the detection redundancy. The simulation verification shows that the method can effectively complete the fault detection of the aircraft control system, and the fault detection rate can be $98.7 \%$.

\section{Negative Selection Algorithm Fault Detection Principle}

There are many artificial immune algorithms, in which the negative selection algorithm proposed by Forrest et al. [14] has been successfully used in the field of fault detection [15, 16]. The negative selection algorithm is inspired by the mechanism of $\mathrm{T}$ cell production in the immune system. It simulates the tolerance process of mature human cells in the thymus and effectively recognizes nonself antigens by generating mature detectors, which has wide application prospects in the field of fault detection. And according to this mechanism, compared with other intelligent algorithms, it requires less prior knowledge, and it further requires less fault samples in the field of fault detection [7, 17].
According to different matching rules and affinity calculation methods, the negative selection algorithm widely applied can be divided into real-value based negative selection algorithm (Real Negative Selection Algorithm, RNSA) proposed by Dasgupta and Forrest [15] and variable radius negative selection algorithm (V-detector), both use ndimensional real vector space as the system state space, and determine the similarity of antibody and antigen by methods such as Euclidean distance. V-detector is an extension of RNSA, which dynamically adjusts the detector radius based on RNSA.

The state space of the system is divided into self-space and non-self-space under the negative selection; the selfspace contains normal samples, and the non-self-space contains the abnormal state samples. At the same time, a large number of candidate detectors with the function of observation are randomly generated, and let these candidate detectors are learned and trained to observe each state sample in the self-state space. Only the candidate detectors that are not sensitive to all the self-states can be retained and become mature detectors, which can participate in the final detection stage, and the flow chart is shown in Figure 1.

\section{The Fault Detection Method of Aircraft Control System}

3.1. Generation of the Mature Aircraft Control System Detectors. Based on the fault detection model above, the fault detection of the aircraft control system can be carried out as follows.

3.1.1. Establish the Control System State Space. The control system state space is composed of a complete set of $\mathrm{N}$ dimensional real-valued normalized vectors $U=[0,1]^{n} ; U$ is all possible system states. The system state is represented by the $\mathrm{N}$-dimensional normalized feature vector $u, u=\left[u_{1} \cdots\right.$ $\left.u_{n}\right] \in U$, and $u_{1}, u_{2}, u_{3}, \cdots u_{n}$, respectively, represent the values of different characteristic parameters, such as the measured values of the main hydraulic pressure, the trailing edge flap deflection angle, and the aileron deflection angle. In the $\mathrm{N}$-dimensional state space $U^{n}$, the space composed of the system's normal state feature vectors is called the self-space, which is represented by the set $S$, and the space composed of the system's abnormal or fault state corresponding feature vectors is called the non-self-space, represented by the set $\bar{S}$, that is, the complement of S.

3.1.2. Affinity Judgment. Affinity is an important criterion for measuring the quality of the detector. It includes two types; one is the affinity of the detector and the self-space; this affinity characterizes the matching degree of the detector and the self-sample; the other is the affinity between the detector and the detector; this affinity characterizes the degree of similarity between the detectors, which is an important indicator for evaluating the diversity of detectors. Assuming that both $E=\left[\begin{array}{llll}E_{1} & E_{2} & \cdots & E_{4}\end{array}\right]$ and $F=\left[\begin{array}{llll}F_{1} & F_{2} & \cdots & F_{4}\end{array}\right]$ are feature vectors in the state space $U^{n}$ of the aircraft control 


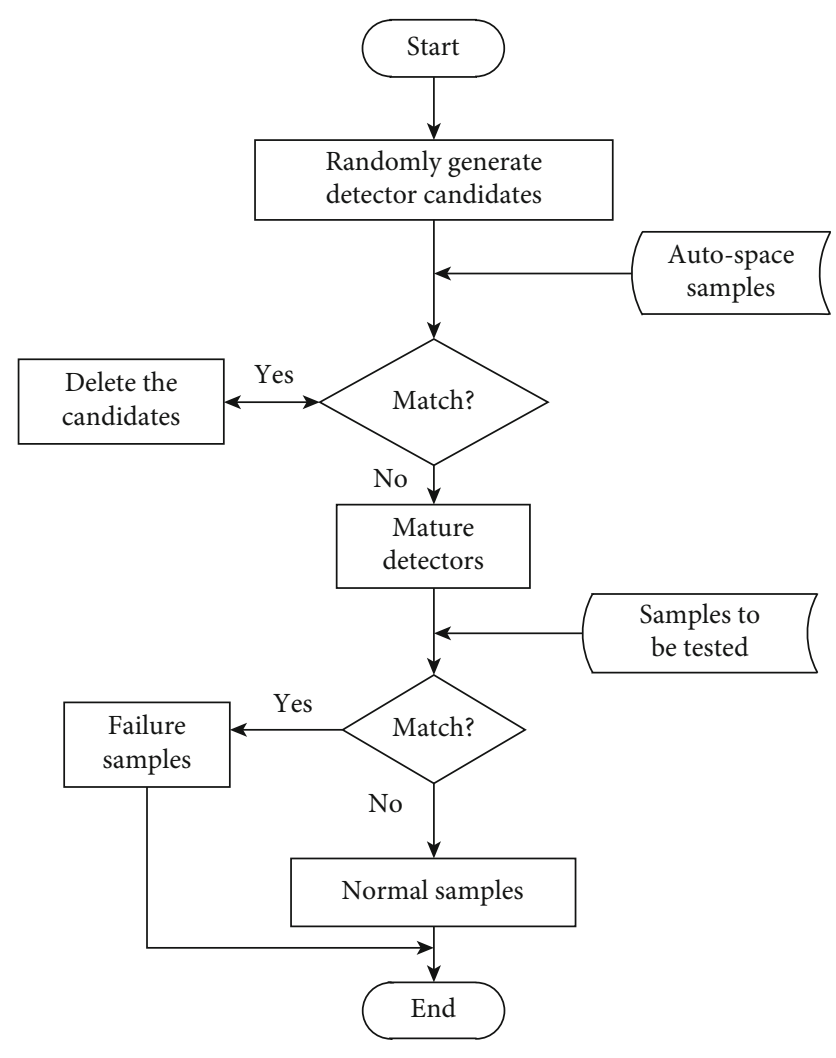

FIGURE 1: Flowchart of negative selection algorithm.

system, the affinity between $E$ and $F$ is often expressed in three ways:

(1) Euclidean distance $d_{1}=\sqrt{\sum_{i=1}^{n}\left(e_{i}-f_{i}\right)^{2}} i=1,2,3, \cdots n$

(2) Manhattan distance $d_{2}=\sum_{i=1}^{n}\left|e_{i}-f_{i}\right| i=1,2,3, \cdots n$.

(3) Chebyshev distance $d_{3}=\max \left|e_{i}-f_{i}\right| i=1,2,3, \cdots n$.

3.1.3. Optimization of the Aircraft Control System Detector. Ideally, fault detection is expected to detect the most faults with the fewest detectors, so the goal of detector optimization is to continuously improve its coverage. The shapes of the detector in the negative selection algorithm are usually hyperrectangle and hypersphere. The latter is more convenient for representation and calculation, which only needs two parameters, center point and radius, to describe it, and has good spatial coverage. Therefore, from these two parameters, the improved method of the hypersphere detector is studied in this paper.

The detector optimization method is shown in Figure 2. Assuming that a randomly generated detector point is $x$ and the two closest self-points in the self-space $S$ to the point $x$ are denoted as $s_{1}$ and $s_{2}$ and the distances from the point $x$ are $L_{1}$ and $L_{2}, L_{1} \leq L_{2}$, that is, $s_{1}$ is the closest self-point to point $x$, and $L_{1}$ is the shortest distance between point $x$ and self-space. In the vector space, the hypersphere with $x$ as the center and $r_{1}=L_{1}-r_{s}$ as the radius is denoted as $B_{1}$, and the hypersphere with $r_{2}=L_{2}-r_{s}$ as the radius is denoted

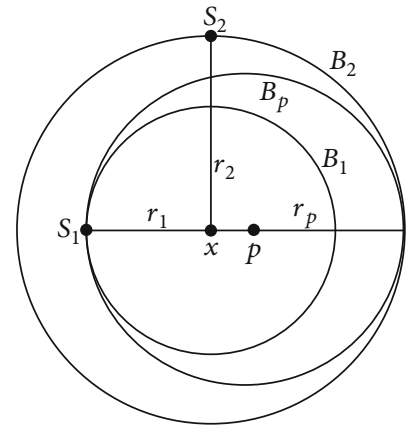

Figure 2: Hypersphere radius optimization diagram.

as $B_{2}$, and $r_{s}$ is the radius of the self-space sample; it is not difficult to get $B_{1}$ included in $B_{2}$. A point $p$ can be obtained on the line connecting $B_{1}$ and point $x$ to make $\left|p-s_{1}\right|=r_{1}+$ $r_{2} / 2$. And taking the point $p$ as the center, the hypersphere with the radius is denoted as $B_{p}$, so $B_{1}$ is included in the hypersphere $B_{p}$, and $s_{1}$ is on the $B_{p}$ sphere. At the same time, $B_{p}$ is included in $B_{2}$, so $B_{p}$ will not cover $s_{2}$. Therefore, the detection range of the detector $\left(p, r_{p}\right)$ includes and is larger than the detection range of the detector $\left(x, r_{1}\right)$, which does not cover points in the self-space. For the reason that the newly generated detector coverage is greater than or equal to the coverage of the original variable radius algorithm.

3.1.4. Determining the Number of Mature Detectors Generated. The random generation of the detector can be regarded as $n$ random samples in non-self-space. For each sampling point, whether the point is covered by the generated detector space, it follows the binomial distribution. According to the central limit theorem, when the number of sample $n$ is large enough, the binomial distribution will approach a normal distribution. So the normal distribution $X \sim N(n p, n p(1-p))$ can be utilized as an approximation to the binomial distribution [18]. On this basis, the hypothesis test method is used to determine the number of detectors that meet the coverage requirements, that is, the hypothesis coverage has reached the expectations and then judge whether the hypothesis is correct. According to the characteristics of the algorithm generated by the detector, the right side unilateral test is used here. The hypothesis test of the algorithm is the original hypothesis $I_{1}$ is the detector coverage, which is lower than the expected value $p_{\min }$, and the alternative hypothesis is the detector coverage $I_{2}$, which is higher than the expected value $p_{\min }$. When $I_{1}$ is true, no more detectors will be added, which will cause the detector coverage to be too low, and when $I_{1}$ is not true, it is necessary to continue to generate detectors, resulting in some overlapping detectors.

In contrast, it is more necessary to avoid the situation where the detector coverage is too low, so the assumptions given here are scientific. According to the above two points, let $p$ be the expected coverage of the detector in non-selfspace and $q$ be the estimated value of the detector coverage obtained by sampling. According to the central limit theorem, so $(q-p /(\sigma / \sqrt{n})) \sim N(0,1) \quad(\sigma \quad$ is the standard 
variance; $n$ is the sample size), and the test statistics $z=$ $(m / n-p) /(\sqrt{p(1-p)} / \sqrt{n})$ ( $m$ is the number of covered points). When $z>z_{\alpha}$ ( $\alpha$ is the significance level), the null hypothesis is rejected, that is, the detector has reached the requirement for the non-self-space coverage of the control system, and the algorithm ends.

3.2. Fault Detection. Enter the sample to be tested and calculate the affinity between it and the mature detector. When the affinity is less than the range of the flap system fault detector, it means that the sample is faulty; otherwise, it is normal. For detection performance, two indicators, failure detection rate $\mathrm{T}$ and false alarm rate $\mathrm{A}$, can be used to measure. The fault detection rate [19] is shown in (1), and the false alarm rate [19] is shown in (2). Where $F_{f}$ is the number of faults detected in the fault sample, $F_{t}$ is the number of faults detected in the normal sample, $T_{t}$ is the number of normal samples detected as normal, and $T_{f}$ is the number of normal samples detected as faults.

$$
\begin{aligned}
& T=\frac{F_{f}}{F_{t}+F_{f}}, \\
& A=\frac{T_{t}}{T_{t}+T_{f}} .
\end{aligned}
$$

\section{Simulation and Analysis of a Certain Type of Aircraft Main Control System Fault Detection}

The flight parameter data of a certain type of the aircraft control system is used to verify the effectiveness of the above fault detection method in this section. The aircraft relies on the control system to control the rudder to maintain a stable flight attitude. And the aircraft control system can generally be divided into two parts: the main control system and the auxiliary control system. The auxiliary control system mainly controls the flap system, including the leading edge slat, trailing edge flap, and spoiler, which is mainly used to improve the maneuverability and climbing ability of the aircraft [20]. The main structure of the aircraft control system is shown in Figure 3. At the same time, the main control system mainly controls left and right ailerons, elevators, and rudders. The aileron control system can be controlled by taking the steering wheel to turn left or right around the steering column and transmitted the motion to the aileron through the transmission system to deflect it up and down, thereby achieving the aircraft horizontal roll in the air. The pilot can control the steering of the rudder left and right by pedaling, thereby controlling the course of the aircraft, and the function of the elevator is to push and pull the steering column to change the aircraft's pitch flight status.

Select 6 kinds of flight parameter data: the input data for the steering column position, pedal position, steering wheel position, and the output data for aileron/elevator/rudder declination, and by fitting the flight data of 134 sorties, the transfer coefficients of the three control channels of aileron, elevator, and rudder can be obtained. The safety analysis report of this type pointed out that the transfer coefficients of the three channels of aileron, elevator, and rudder have corresponding quantitative requirements, when actuator and other devices in the three channels are deformed or damaged, the three coefficients will be changed, and related failure modes can be introduced. Therefore, the transfer coefficients of the three control channels are selected as the characteristic parameters of fault detection and construct the characteristic space. Part of the flight data is shown in Table 1 .

4.1. Preprocess Sample Data. After fitting the above six kinds of flight parameter data, the transfer coefficients of three channels can be obtained. And these coefficients further need to be normalized to obtain dimensionless characteristic parameters. The maximum and minimum normalization method is adopted to normalize here, that is:

$$
x^{\prime}=\frac{x-\min (x)}{\max (x)-\min (x)},
$$

where $x^{\prime}$ is the normalized data, $x$ is the data before normalization, and $\max (x)$ and $\min (x)$ are the maximum and minimum values in the feature vector $x$, respectively.

4.2. Simulation Verification. From the above algorithm flow and test data, it can be seen that the main parameters that affect the test results are affinity judgment method, autoradius, and significance level. The affinity judgment method used in this paper is the European distance method. Although the complexity of the European distance method is relatively high, as the computer runs faster, its accuracy is higher than the other two methods, and it is convenient to judge the randomly generated whether the detector point is within the generated detector, so it is more suitable for the algorithm. In this paper, the experiments verify the fault detection rate, false alarm rate, and the number of detectors generated by the algorithm when the auto-radius is 0.02 to 0.20 and the significance level $\alpha$ is 0.01 to 0.20 to find the best parameters to get the most effective detection method; the results are shown in Figures 4-7.

As for the auto-radius, it can be seen from Figures 4 and 5 that the smaller the auto-radius, the higher the fault detection rate and the fewer the number of detectors generated, but the false alarm rate also increases. The main reason is that when the auto-radius is too small, the self-space is relatively discrete, and partial detectors are generated in the self-space gap, resulting in an increase in the false alarm rate; At the same time, when the self-radius is small, the detection is stricter in the relatively discrete self-space so that more faults can be detected, and the fault detection rate is improved. It should be noted here that when the auto-radius is 0.18 , the number of detectors increases suddenly because of its own image attributes, resulting in many detectors near the selfspace. Therefore, the auto-radius is more appropriate to be taken as 0.05 .

And for the significance level, as can be seen from Figures 6 and 7.The smaller the $\alpha$, the higher the detection rate, but at the same time, the false alarm rate has also increased, and the number of detectors required has also 


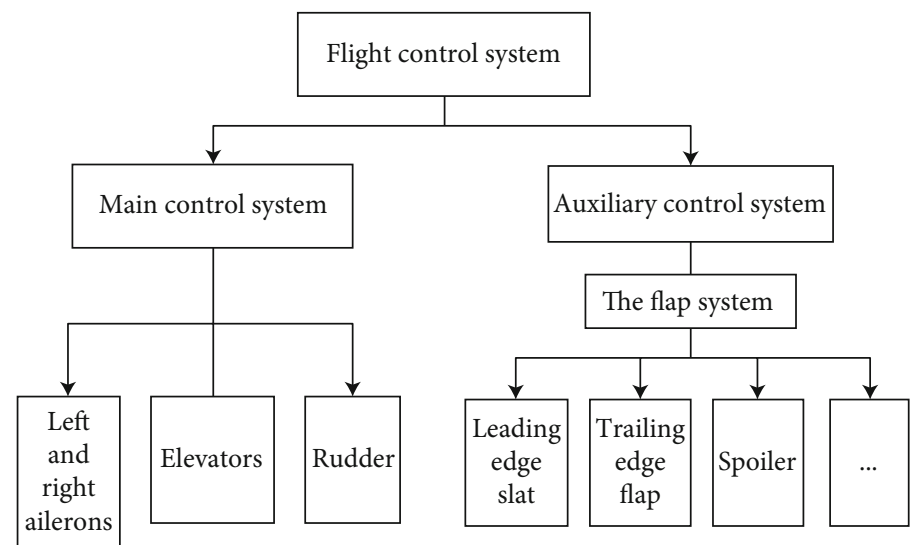

FIgURE 3: The main structure of the aircraft control system.

TABle 1: Aircraft flight parameter data (partial).

\begin{tabular}{lccccc}
\hline $\begin{array}{l}\text { Steering column } \\
\text { position } / \mathrm{mm}\end{array}$ & $\begin{array}{c}\text { Pedal } \\
\text { position } / \mathrm{mm}\end{array}$ & $\begin{array}{c}\text { Steering wheel } \\
\text { position }{ }^{\circ}\end{array}$ & $\begin{array}{c}\text { Aileron } \\
\text { declination }{ }^{\circ}\end{array}$ & $\begin{array}{c}\text { Rudder } \\
\text { declination }{ }^{\circ}\end{array}$ & $\begin{array}{c}\text { Elevator } \\
\text { declination }{ }^{\circ}\end{array}$ \\
\hline Front 39.180 & Front 0.670 & Left 4.606 & Down 0.996 & Left 0.352 & Down 6.738 \\
Front 38.219 & Front 0.670 & Left 4.222 & Down 1.055 & Left 0.352 & Down 6.738 \\
Front 38.219 & Front 0.670 & Left 4.222 & Down 1.055 & Left 0.352 & Down 6.738 \\
Front 38.219 & Front 0.670 & Left 4.222 & Down 0.996 & Left 0.352 & Down 6.738 \\
Front 38.219 & Front 0.670 & Left 4.222 & Down 1.055 & Left 0.352 & Down 6.738 \\
Front 38.219 & Front 0.670 & Left 4.222 & Down 1.055 & Left 0.352 & Down 6.738 \\
Front 38.219 & Front 0.670 & Left 4.222 & Down 1.055 & Left 0.352 & Down 6.738 \\
\hline
\end{tabular}

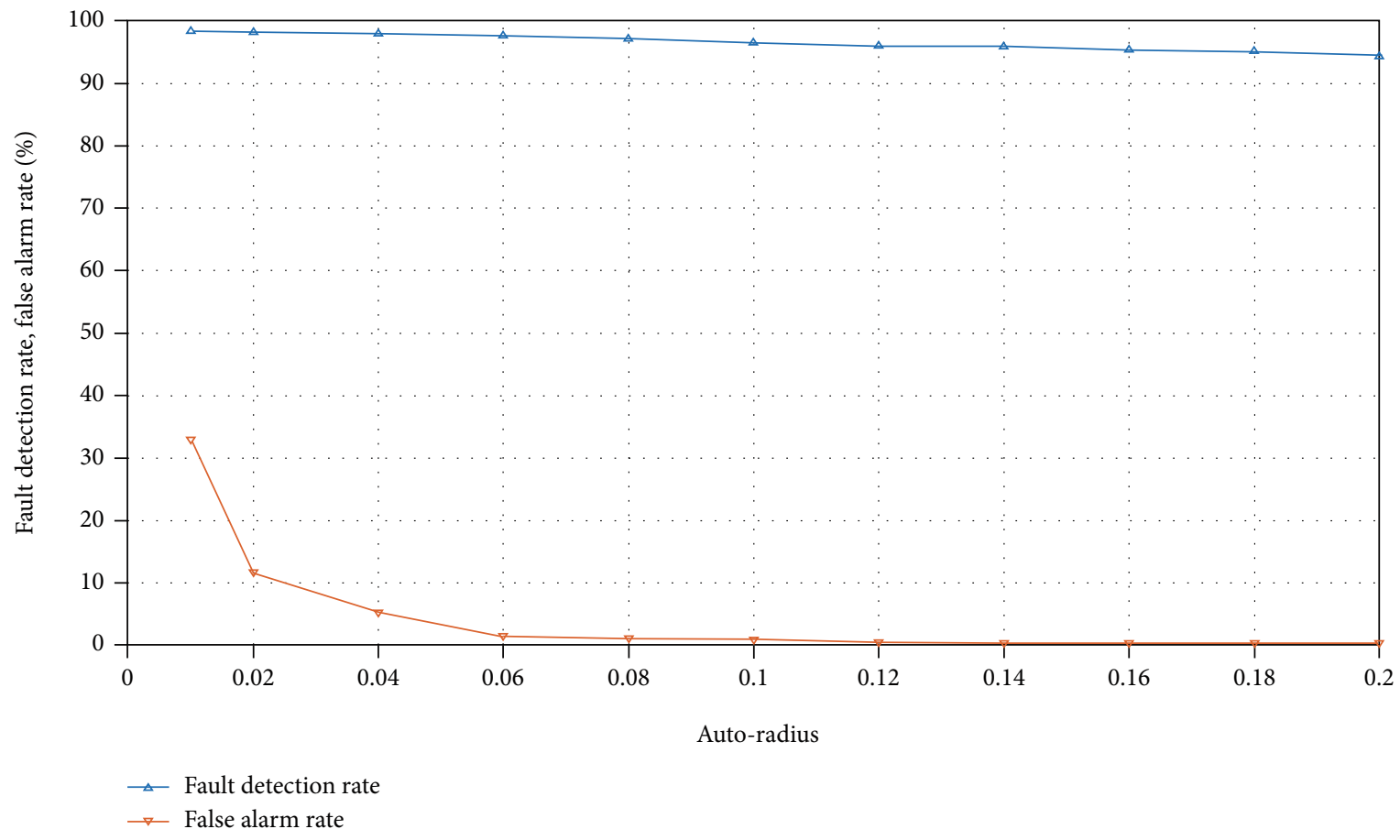

FIgURE 4: Effect of self-space auto-radius on fault detection rate and false alarm rate. 


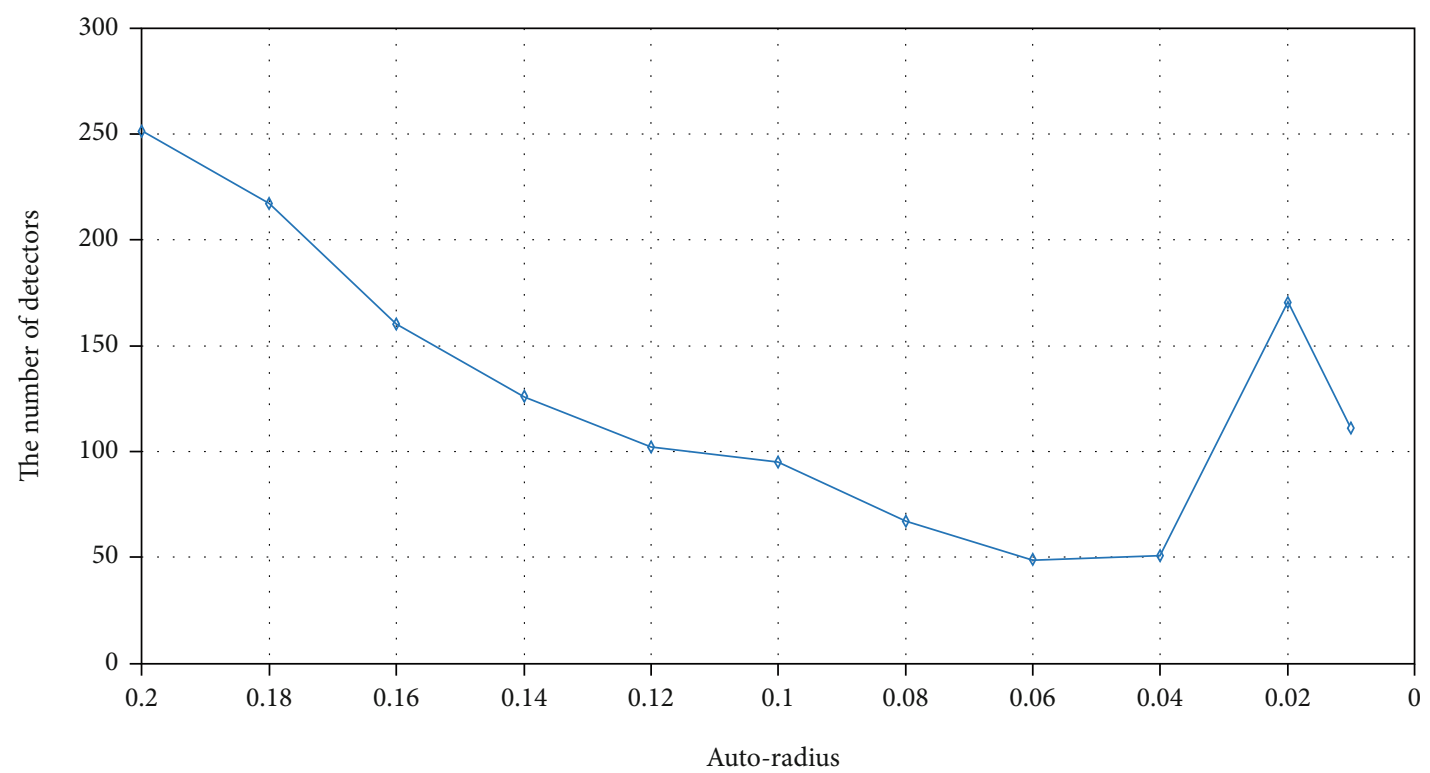

Figure 5: Effect of self-space auto-radius on the number of detectors.

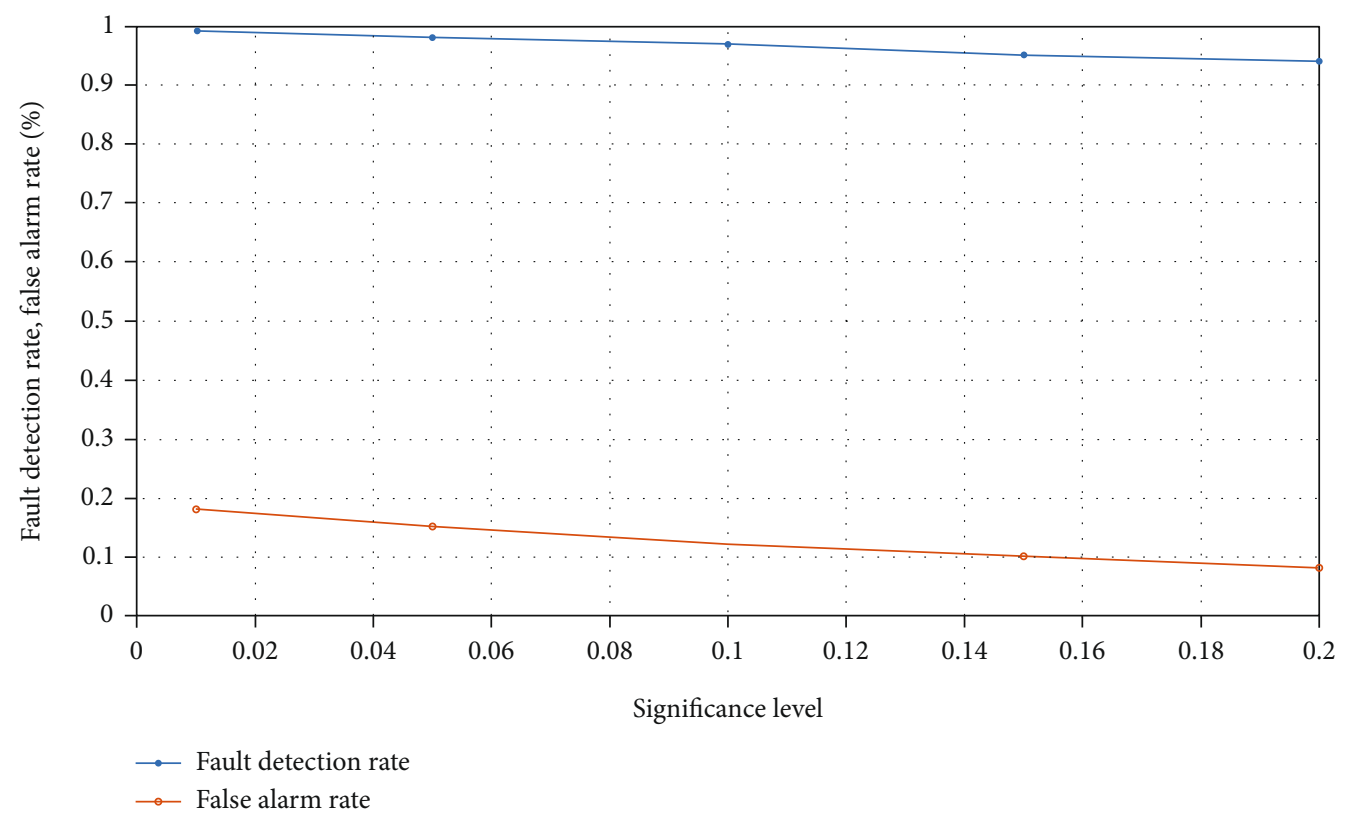

FiguRE 6: Effect of significance level on fault detection rate and false alarm rate.

increased. According to statistical theory, when the significance level is smaller, the probability that the detector coverage rate does not reach the expected value will be smaller, and the probability that the redundant detector will increase will become larger, which shows that the experimental results are consistent with the theoretical analysis. Combining both of them, $\alpha$ is more appropriate to be taken as 0.1 .

Select the parameter of the algorithm, detector expected coverage $p=0.995$, auto-radius $r_{s}=0.05$, significance level $\alpha=0.1$, and then, it can be obtained $z_{\alpha}=1.65$ according to the normal distribution. Under this condition, the fault detection rate of the aircraft main control system is $98.3 \%$, the false alarm rate is $1.7 \%$, and the number of detectors is 54; the detection performance is excellent.

The self-space, detector space, and fault space generated by the algorithm are shown in Figures 8 and 9,where Figure 8 is the self-space composed of the transfer coefficients of the three control channels (the aileron, elevator, and rudder), and Figure 9 is the self-space (purple), the detector space generated (yellow) from the self-space, and the fault sample space (black spot). In Figure 9(a), due to the detector space is like a hollow ball and the angle of view, it is not clear that all the fault samples fall in the detector space, but when the coordinate axis is adjusted, 


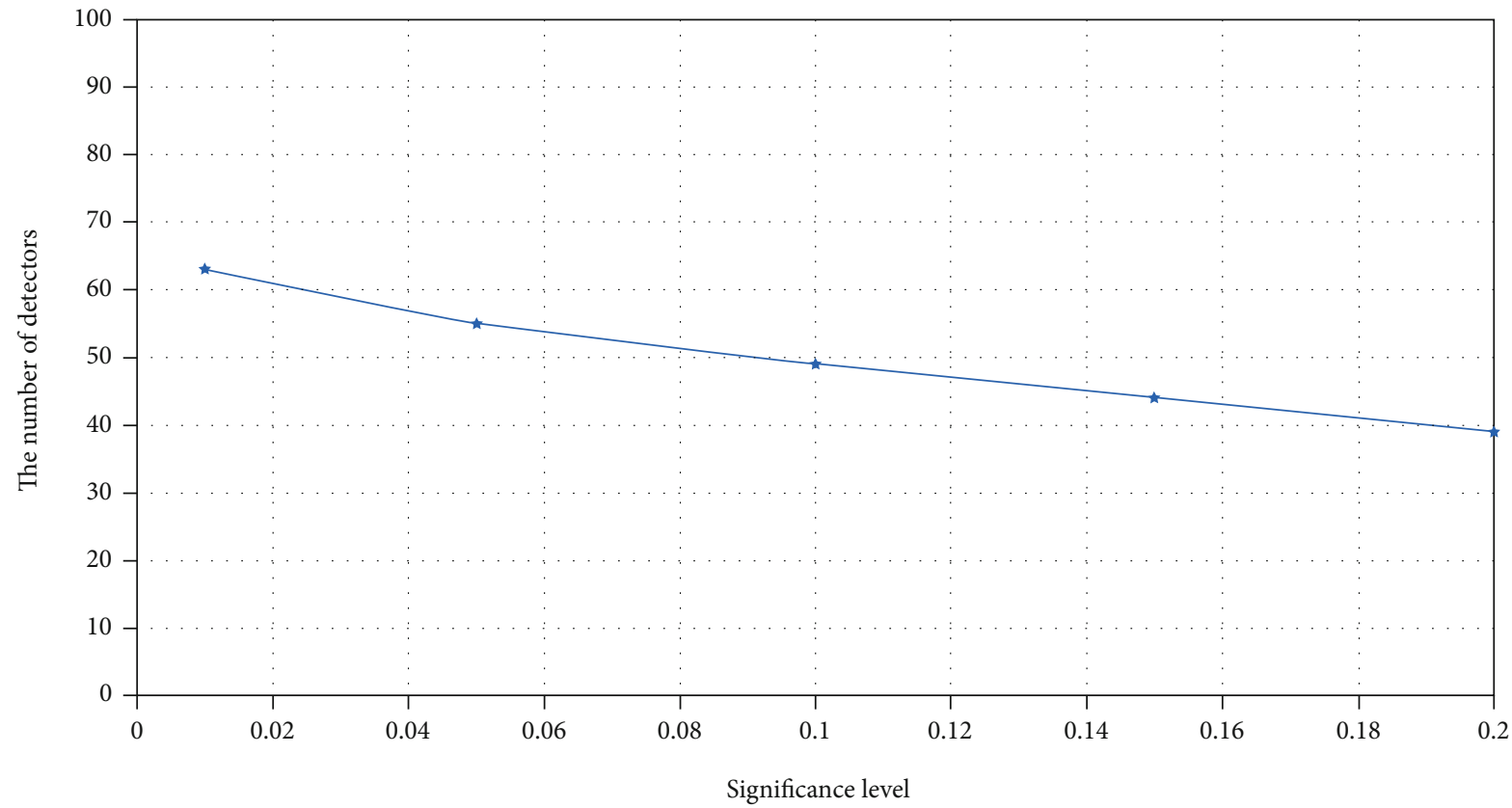

Figure 7: Effect of significance level on the number of detectors.

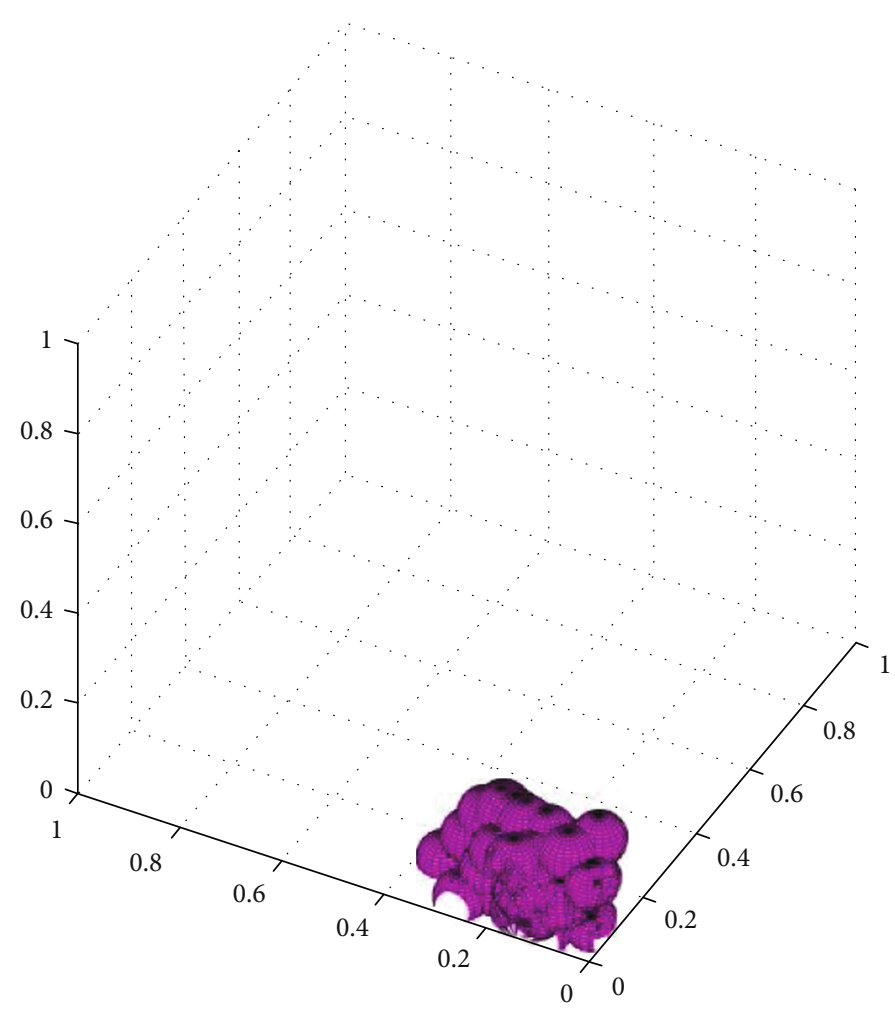

Figure 8: Self-space.

we can see that the detector space covers all the fault samples completely in Figure 9(b), reflecting that the method has a good detection effect.

Because the two-dimensional plane can better show the generation effect of the detector, the two-dimensional data of the rudder (ordinate) and elevator (abscissa) are selected to simulate the detector generation process of the three algorithms in this paper. The results are shown in Figure 10 (RNSA), Figure 11 (V-detector), and Figure 12 (algorithm in this paper). By contrast, the number of detectors generated by the optimized negative selection algorithm is greatly reduced. 


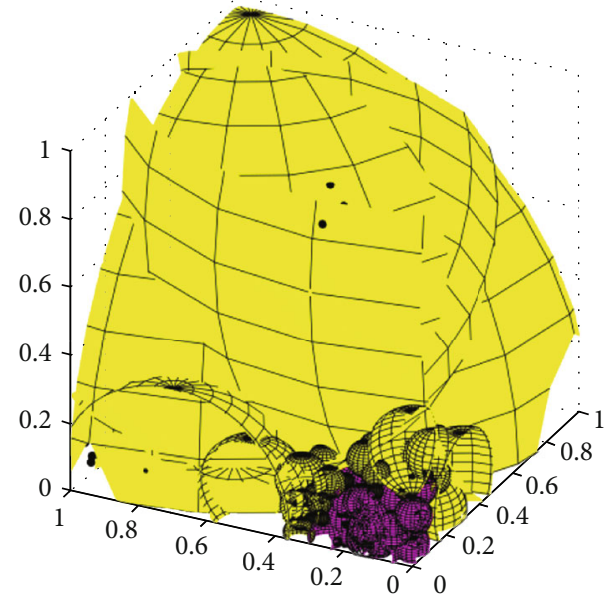

(a)

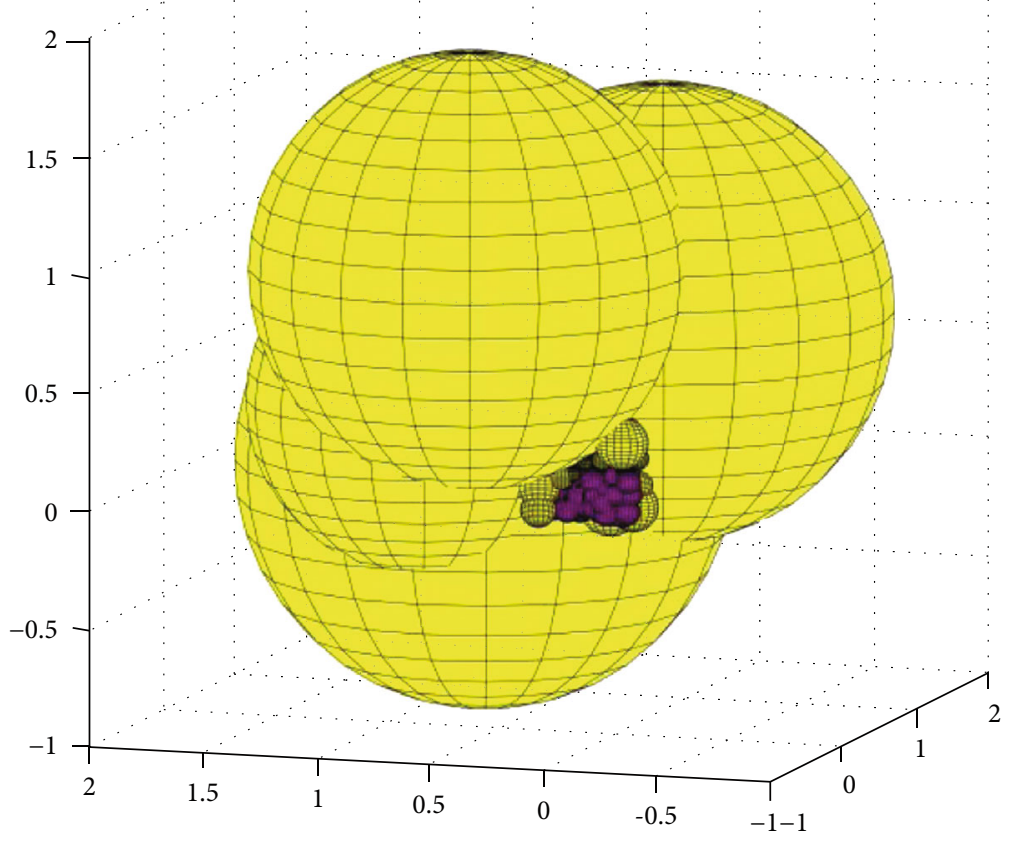

(b)

FIgURE 9: Self-space, detectors space, and fault sample space.



Figure 10: Detectors generation of RNSA.



Self-space detectors

Figure 11: Detectors generation of V-detector.
In order to further reflect the superiority of the optimized algorithm, two traditional negative selection algorithms (RNSA and V-detector) and the algorithm proposed in this paper were simulated and compared, and the simulation results are shown in Table 2. It can be seen that compared with the traditional negative selection algorithm, fewer detectors are generated by the optimized algorithm, and the fault detection effect is better.

\section{Conclusion}

In this paper, a new approach is presented for the fault detection of aircraft control system with the help of negative selection artificial immune system, and which is verified by real QAR data. The geometric mathematics is utilized to improve the overall coverage of the detector and reduce the coincidence between detectors, as well as the hypothesis test is used 


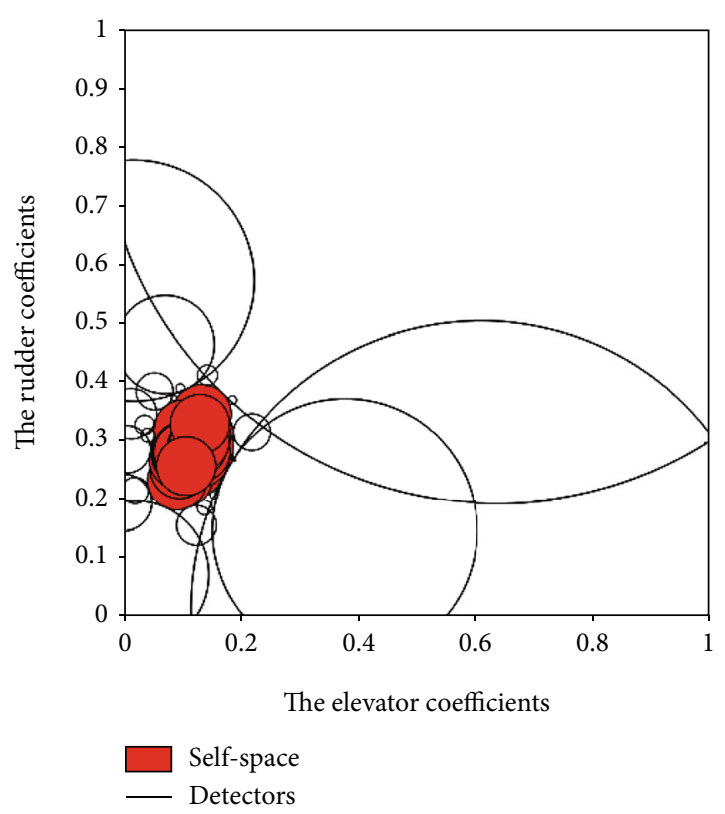

FIGURE 12: Detectors generation in this paper.

TABLE 2: The number of detectors, detection rate, and false alarm rate of three algorithms.

\begin{tabular}{lccc}
\hline Algorithm & $\begin{array}{c}\text { The number of } \\
\text { detectors }\end{array}$ & $\begin{array}{c}\text { Fault } \\
\text { detection } \\
\text { rate/\% }\end{array}$ & $\begin{array}{c}\text { False } \\
\text { alarm } \\
\text { rate/\% }\end{array}$ \\
\hline RNSA & 532 & 91.4 & 16.3 \\
$\begin{array}{l}\text { V-detector } \\
\begin{array}{l}\text { The proposed } \\
\text { method in this paper }\end{array}\end{array}$ & 541 & 93.2 & 8.9 \\
\hline
\end{tabular}

as the test standard of coverage, which made the generated detector more streamlined. With the advancement of aviation technology, the reliability and safety of aircraft are getting higher and higher, and it leads to the reduction of fault data. In the field of fault detection, compared with intelligent algorithms such as neural network and clustering which need mass of fault data to improve the accuracy of fault detection, negative selection requires less prior knowledge and fault data so its superiority is more and more prominent.

The case proves that the fault detection method is feasible and efficient, and the authors believe that this work will contribute to the field of aircraft control system PHM.

\section{Data Availability}

The data used to support the findings of this study have not been made available because the data come from a research institute, and we both have a confidentiality agreement with each other that the data cannot be shared.

\section{Conflicts of Interest}

The authors declare that there are no conflicts of interest regarding the publication of this paper.

\section{Acknowledgments}

This work is supported by the National Natural Science Foundation of China (Grant no. 61873203), the Natural Science Basic Research Plan in Shaanxi Province of China (program No. 2019JM-003), and the Research funds for interdisciplinary subject, NWPU, and key laboratory fund (6142806190504).

\section{References}

[1] Z. N. Jiang, The research of health assessment fault diagnosis method of civil aircraft flight control system, Nanjing University of Aeronautics and Astronautics, Nanjing, 2018.

[2] R. H. Xu and J. B. Sun, "Fault diagnosis for the fly-by-wire system based on artificial network and expert system," Chinese Journal of Aeronautics, vol. 26, no. 2, pp. 195-198, 2005.

[3] Z. B. Duan and P. Zhang, "Fault diagnosis method of flight control system based on ontology and FMECA," Journal of Civil Aviation University of China, vol. 34, no. 4, pp. 21-26, 2016.

[4] K. Cheng, Aircraft control system condition monitoring and fault forecast method research, Nanjing University of Aeronautics and Astronautics, Nanjing, 2014.

[5] G. C. Luh and W. C. Cheng, "Immune model-based fault diagnosis," Mathematics and Computers in Simulation, vol. 67, no. 6, pp. 515-539, 2005.

[6] H. W. Mo, The principles and applications of artificial immune system, Harbin Institute of Technology Press, Harbin, 2003.

[7] Y. H. Liu, The Rresearch of Aanomaly Ddetection and Ffault diagnosis Based on artificial immune system, Shanghai University, Shanghai, 2013.

[8] C. A. Laurentys, R. M. Palhares, and W. M. Caminhas, "Design of an artificial immune system based on danger model for fault detection," Expert Systems with Applications, vol. 37, no. 7, pp. 5145-5152, 2010.

[9] K. Ghosh and R. Srinivasan, "Immune system in spired approach to process monitoring and fault diagnosis," Industrial and Engineering Chemistry Research, vol. 50, no. 3, pp. 1637-1651, 2010.

[10] F. Diego, A. E. Garcia, and H. M. Perez, "Spacecraft heath monitoring using a biomimetic fault diagnosis scheme," Journal of Aerospace Information Systems, vol. 15, no. 7, pp. 396413, 2018.

[11] J. G. Sha, The research on fault diagnosis of aeroengine digital control system based on artificial immune theory, Nanjing University of Aeronautics and Astronautics, Nanjing, 2007.

[12] A. X. Ma and Y. J. Li, "Intelligent diagnosis for aircraft engine wear fault based on immune theory," Chinese Journal of Aeronautics, vol. 36, no. 6, pp. 1896-1904, 2015.

[13] Y. K. Zhang, "Fault diagnosis of airborne electro-mechanical systems based on immune algorithm," Electronic Science and Technology, vol. 28, no. 6, pp. 83-85, 2015.

[14] S. Forrest, A. S. Perelson, L. Allen, and R. Cherukuri, "Selfnonself discrimination in a computer," in 2012 IEEE Symposium on Security and Privacy, vol. 202, Piscataway, NJ, 1994.

[15] D. Dasgupta and S. Forrest, "Artificial immune systems in industrial applications," in Proceedings of the Second International Conference on Processing and Manufacturing of Materials, vol. 1, pp. 257-267, Honolulu, HI, USA, 1999. 
[16] M. J. Romer, E. O. Nwadiogbu, and G. Bloor, "Development of diagnostic technologies for aerospace health management applications," in IEEE Proceedings of Aerospace Conference, vol. 6, pp. 3139-3147, Piscataway, NJ, 2001.

[17] C. J. Liu, Y. Li, and Y. P. Wang, "Optimization and application of real-valued negative selection algorithm," Computer Measurement \& Control, vol. 20, no. 12, pp. 3158-3164, 2012.

[18] Y. M. Shi, W. Xu, C. Y. Qin, and Y. Xu, Mathematical Statistics, Science Press, Beijing, 4th edition, 2015.

[19] G. Vachtsevanos, F. Lewis, M. Roemer, A. Hess, and B. Wu, Intelligent Fault Diagnosis and Prognosis for Engineering Systems, Wiley, New York, 2007.

[20] Z. T. Wu and X. R. Li, "Fault evaluation method of aircraft control system based on flight data," Computer Measurement \& Control, vol. 27, no. 7, pp. 275-279, 2019. 